\title{
Analysis of Long-Term Weight Regain in Obese Patients Treated with Intragastric Balloon
}

\section{Bruno Queiroz Sander*, Luiz Ronaldo Alberti, Diogo Turiani Hourneaux Moura, Jimi Izaques Bifi Scarparo, Patricia Sanches Carneiro, Vitor Nunes Arantes}

Department of Surgery, Faculty of Minas Gerais - Medical Science, Brazil

*Corresponding Author: Bruno Queiroz Sander, Department of Surgery, Faculty of Minas Gerais - Medical Science, Brazil.

Received: October 09, 2019; Published: November 04, 2019

DOI: $10.31080 /$ ASGIS.2019.02.0094

\begin{abstract}
Background and Aims: Obesity is a global disease and its management includes pharmacological therapy, surgery and Intragastric Ballon (IGB). IGB has gained popularity recently, however, studies addressing long-term weight loss IGB are scare in the current literature. This study aimed to assess the weight regain in a large cohort of patients treated with IGB. The objective is to communicate the results based on our experience with the percutaneous treatment of this complication.

Methods: Patients treated with IGB for a six-month, who underwent ballon removal at least two years before the collection of data, were invited to participate in the study. Patients were interviewed and medical records were analyzed, after were stratified based in length of follow-up after IGB removal on date of interview (2, 3 and 4 years).

Results: During the use of IGB patients lost a mean of $66 \%$ of excess weight. Between 2 and 4 years after removal of IGB $67 \%$ of the subjects had regained weight. The correlation between the BMI scores and the weight regain was inversely $(r=-0.20)$ and significant $(\mathrm{p}<0.01)$.

Conclusion: The percentage of weight regained after treatment for obesity with IGB is high but within the standards described in the literature.

Keywords: Intragastric Balloon; Weight Gain; Obesity; Endoscopy
\end{abstract}

\section{Introduction}

Obesity is a recurrent, epidemic and growing health problem, and its management remains a challenge. Obesity can traditionally be treated through different combination of programs involving diet control, exercises, behavioral and psychological counseling, in addition to appetite-control drugs. However, therapeutic failure of these measures are high, and even initially successful cases still show that weight maintenance over a long-term period frequently fails [1-3]

Recently, the intragastric balloon (IGB) has become a popular alternative to manage obese patients, because it is a relatively safe and minimally invasive endoscopic method that has been recognized by the National Institutes of Health (NIH) [4] and FDA as an option to manage obese individuals potentially reducing mortality [5]. In Brazil, the IGB is indicated for patients with BMI equal or greater than $27 \mathrm{~kg} / \mathrm{m}$.

Many studies [1,4-8] show successful weight loss after the use of IGB for approximately 6 months. However, no studies have reported long-term follow-up concerning weight regain among these patients after removal of the IGB. This study aimed to assess the long-term weight regain in a large cohort of patients treated with
IGB, with a time span from two to five years after the removal of the device.

\section{Methods}

Ethical aspects and delineation

This study was approved by the Ethical and Research Committee (Brazilian equivalent to an Internal Review Board) of Federal University of Minas Gerais (CAE number 42995915.4.0000.5132), as part of the postgraduate program in Adult Health Sciences. All eligible participants signed the consent form.

The subjects were selected from a population treated at a private obese outpatient medical center. All patients were contacted by phone and invited to participate in the study. The inclusion criteria in the study were: use of IGB for at least 5 months, no bariatric surgical procedure after removal of the IGB and no history of pregnancy after IGB removal.

We analyzed the medical data recorded during the whole follow-up period, as well as a semi-structured interview was personally obtained by trained professionals for physical anamnesis and qualitative evaluation of the treatment. A total of 219 patients were recruited and divided into groups according to post-discharge follow-up time, 2 years $(n=28)$, 3 years $(n=114)$ and 4 years $(n=77)$. 


\section{Concepts and procedures}

In the study, the term "comorbidities" was defined as presence of metabolic or respiratory diseases. The term "diagnosis of a disease" refers to any disease related to obesity. The term "undergone a surgery" refers to any kind of surgery, except bariatric ones. The term "sedentary" refers to patients who did not practice any kind of physical activity on any day of the week. The term "use of medicines" refers to patients who made use of medication to control chronic diseases such as diabetes and arterial hypertension.

\section{Statistical analysis}

Data were analyzed using Stata statistical package (version 13.0). Comparisons of explicative variables concerning the positive or negative group of weight regain were performed by using the chi-squared test for qualitative variables and the Student $t$ test for quantitative variables. All associations were considered significant at $\mathrm{p} \leq 0.05$.

\section{Results}

It is a predominantly female sample (81.2\%), of young adults (mean age: $37.29 \pm 9.07$ years), with no significant difference between the mean age according to the annalistic groups ( $\mathrm{p}=0.56)$. During the use of IGB, patients lost an average of $15 \%$ of their total body weight; representing a mean of $66 \%$ of excess weight loss (the mean weight loss during treatment was $15.3 \pm 6.9 \mathrm{~kg}$ ). Between 2 and 4 years after removal of IGB, $67 \%$ of the subjects had regained weight ( 2 years: $4.66 \pm 4.91 \mathrm{~kg}$; 3 years: $8.66 \pm 6.97 ; 4$ years: $9.99 \pm 8.44$ ). Most patients $(62 \%)$ regained $10 \%$ to $19 \%$ of total weight loss during treatment (Table 1).

\begin{tabular}{|l|c|c|c|}
\hline \multirow{2}{*}{ \% weight regain } & & Follow up & \\
\cline { 2 - 4 } & 2 years & 3 years & 4 years \\
\hline$<10 \%$ & 20 & 15,6 & 18,5 \\
\hline Between 10 e 19\% & 70 & 62,7 & 59,3 \\
\hline Between 20 e 29\% & 10 & 14,5 & 14,8 \\
\hline Between 30 e 39\% & & 2,4 & 1,9 \\
\hline Between 40 e 49\% & & 1,2 & 5,6 \\
\hline Between 50 e 59\% & & 2,4 & \\
\hline Between 90 e 99\% & & 1,23 & \\
\hline
\end{tabular}

Table 1: Total weight loss during treatment.

A higher percentage of individuals with a weight regain was observed for those who started BMI $<30 \mathrm{~kg} / \mathrm{m}^{2}$ when compared to BMI scores (BMI<30 kg/m: 71.4\%; $30-40 \mathrm{~kg} / \mathrm{m}$ : 67.1\%; $40-50$ $\mathrm{kg} / \mathrm{m}: 62.9 \%$; > $50 \mathrm{~kg} / \mathrm{m}$ : 50\%), but with no difference in followup times. The correlation between the BMI scores and the weight regain was inversely $(r=-0.20)$ and significant $(p<0.01)$ (Figure $1-\mathrm{A})$. The correlation was stronger and more significant with patients with a 2year follow-up ( $r=-0.59, \mathrm{p}<0.01$ ) (Figure 1-B) followed by the 4-year follow-up sample $(r=0.23 ; p=0.03)$ (Figure $9-\mathrm{C}$ ). For the 3-year follow-up group, the correlation was weak and nonsignificant $(r=-0.06, p=0.53$ ) (Figure 9-D).

\section{Discussion}

To our knowledge, this study is the largest population of patients treated with IGB aiming to investigate long-term efficacy of this device in overweight and obese patients.

Studies about long-term weight regain in patients who underwent an IGB procedure are still limited in the literature $[1,4-7,9,10]$, many studies report on the failure of isolated treatments such as diet, physical exercise and bariatric surgery or even a combination of these interventions [3,11-13]. Those procedures are efficient for weight loss during treatment, but weight regain is prevalent after all these interventions. IGB also can result in a transitory effect if the patient is not involved in re-education about diet and life style modifications. It is important to emphasize that permanent medical care should not be restricted to the period of IGB use, and that treatment must provide conditions for patients to lose weight, receive re-education about their diet habits and improve their health status so that, after IGB removal, the patients be prepared to benefit and maintain the weight reached.

The peak increase in weight regain occurred within 2 years after treatment; it practically doubled in the third year and stabilized in the subsequent year. This fact shows that the first 2 years are of paramount importance to avoid weight regain. One possible explanation is the social environment acquired after treatment. Weight loss gives back to patients a social environment that favors an increased caloric consumption.

It is important to note that weight regain in $53 \%$ of the sample of the study remained between $10 \%$ and $20 \%$ of the percentage of weight lost during treatment. Less than $1 \%$ of the sample regained all the weight lost, and no patients had weight regain greater than the weight lost during treatment. Also add the fact that although $66 \%$ of the total sample had some weight regain, this regrowth was $30 \%$ of the weight lost during the treatment, showing a promising long-term result. In studies describing long-term results of patients undergoing bariatric surgery for obesity, there are significant reports of weight regain like in a study conducted by Catalano., et al. [14], after 18 years on average of follow-up, patients had regained $28 \mathrm{~kg}$.

There is no consensus in the literature regarding the minimum BMI for the patient to be admitted as eligible for the procedure. Based on national studies, each institution set a cutoff point.

Thus this work contributes to establishing the fundamentals of obesity treatment guidelines with IGB. The data presented indicate that patients with $\mathrm{BMI}<30 \mathrm{~kg} / \mathrm{m}^{2}$ do not benefit in the long term compared to weight regain regimen, when compared with patients with BMI> $30 \mathrm{~kg} / \mathrm{m}^{2}$. Studies with metabolic approaches need to be performed to identify the likely causes of increased weight regain 
in this group and other methods of weight loss should be prioritized whenever possible.

When there is consensus that these variables are involved in different ways in weight regain, it is necessary to determine and measure risk for each variable in order to support new tools and follow-up strategies for treatment of obesity in patients who used IGB.

This study has limitations associated with its investigation process. First, this sample was analyzed during a 2- to 4-year followup period; however, we could not measure the weight gain in parametric form on the sample. This has implications because time and variation of long-term weight regain have an impact in such a way that data extrapolation must consider these issues.

We conclude that the percentage of weight regained after treatment for obesity with IGB is high but within the standards described in the literature; however, the percentage of weight regained in this sample was, in most cases, between $10 \%$ and $20 \%$ of weight lost during treatment.

\section{Acknowledgements}

For the great help during the data collection, we thank the medical students of the Medical School of the University of Minas Gerais: Maria Inês Stefanini Zamae; Ramon Meira Assis; Matheus Caldas Avelar and Lucas Lima Aguiar.

\section{Bibliography}

1. Khan Z., et al. "Efficacy of Endoscopic Interventions for the Management of Obesity: a Meta-analysis to Compare Endoscopic Sleeve Gastroplasty, AspireAssist, and Primary Obesity Surgery Endolumenal". Obesity Surgery (2019).

2. Nudel J and Sanchez VM. "Surgical management of obesity". Metabolism: Clinical and Experimental 92 (2019): 206-216.

3. Petridou A., et al. "Exercise in the management of obesity". Metabolism: Clinical and Experimental 92 (2019): 163-169.

4. Al-Momen A and El-Mogy I. "Intragastric balloon for obesity: a retrospective evaluation of tolerance and efficacy". Obesity surgery 15 (2005): 101-105.

5. Genco A., et al. "Intragastric balloon followed by diet vs intragastric balloon followed by another balloon: a prospective study on 100 patients". Obesity Surgery 20 (2010): 1496-1500.

6. Ponce J., et al. "Prospective, randomized, multicenter study evaluating safety and efficacy of intragastric dual-balloon in obesity". Surgery for obesity and related diseases 9 (2013): 290-295.
7. Genco A., et al. "Intragastric balloon for obesity treatment: results of a multicentric evaluation for balloons left in place for more than 6 months". Surgical endoscopy 29 (2015): 23392343.

8. Abeid M., et al. "Efficacy and Safety of Intragastric Balloon Placements in 1600 Case, an Experience from the Middle East". Obesity surgery (2019).

9. Al-Sabah S., et al. "Efficacy of intragastric balloon for the management of obesity: experience from Kuwait". Surgical Endoscopy 30 (2016): 424-429.

10. Jamal MH., et al. "The Safety and Efficacy of Procedureless Gastric Balloon: a Study Examining the Effect of Elipse Intragastric Balloon Safety, Short and Medium Term Effects on Weight Loss with 1Year Follow-Up Post-removal". Obesity Surgery 29 (2019): 1236-1241.

11. Byrne SM., et al. "Psychological predictors of weight regain in obesity". Behaviour Research and Therapy 42 (2004): 13411356.

12. Rogge MM and Gautam B. "Biology of obesity and weight regain: Implications for clinical practice". Journal of the American Association of Nurse Practitioners 29 (2017): S15S29.

13. Sainsbury K., et al. "Attribution of weight regain to emotional reasons amongst European adults with overweight and obesity who regained weight following a weight loss attempt". Eating and Weight Disorders: EWD 24 (2019): 351361.

14. Catalano MF., et al. "Weight gain after bariatric surgery as a result of a large gastric stoma: endotherapy with sodium morrhuate may prevent the need for surgical revision". Gastrointestinal endoscopy 66 (2007): 240-245.

\section{Volume 2 Issue 10 December 2019 (C) All rights are reserved by Bruno Queiroz Sander., et al.}

